\title{
Efficient RFID-Based Mobile Object Localization
}

\author{
Kirti Chawla \\ Department of Computer Science \\ University of Virginia \\ Charlottesville, VA, 22904, USA \\ kirti@virginia.edu
}

\author{
Gabriel Robins \\ Department of Computer Science \\ University of Virginia \\ Charlottesville, VA, 22904, USA \\ robins@virginia.edu
}

\author{
Liuyi Zhang \\ Department of Computer Science \\ University of Virginia \\ Charlottesville, VA, 22904, USA \\ lz3m@virginia.edu
}

\begin{abstract}
Location-awareness of mobile objects is the key to numerous emerging ubiquitous computing applications. We show that RFID technology can be leveraged to achieve mobile object localization in an inexpensive, power efficient, scalable, widely applicable, flexible, and user-friendly manner. We outline the challenges that can adversely affect RFID-based localization techniques, and propose solutions to mitigate them. We present several algorithms for RFID-based mobile object localization that compare favorably or exceed previous methods in terms of accuracy, speed, reliability, scalability, and cost.
\end{abstract}

Keywords - RFID, Object localization, RFID-based positioning

\section{INTRODUCTION}

The confluence of radio frequency identification (RFID) and other wireless technologies lies at the heart of many emerging applications, such as remote medicine, robotic teams, wireless sensing, early warning systems (e.g., for tsunamis, earthquakes, and chemical spills), locating points of interests (e.g., ATMs, banks, and hospitals), and automated inventory management $[1,2,9,10,13,15,16,17,24]$. Such applications require capabilities that include object identification, real-time object tracking, and position localization.

While typical RFID technology is sufficient for object tracking and identification, it does not normally provide object localization capabilities. Several RFID-based localization techniques for mobile objects have been proposed $[5,8,11$, 12]. However, these localization techniques tend to compromise key requirements such as accuracy, speed, power, cost, scalability, and reliability, which severely degrade the utility of these methods. Moreover, some previous localization methods also require cumbersome non-RFID technologies such as ultrasonic sensors, vision sensors, cameras, etc.

We propose to address these limitations by developing a scalable and reliable RFID-based localization approach that accurately and quickly determines the positions of mobile objects. Our approach consists of two separate techniques to localize target tags, as well as localize readers attached to mobile objects. To localize mobile target tags, we vary the reader power levels over a set of calibrated reference tags having known sensitivities. Separately, we determine the positions of target mobile readers by measuring their proximity to reference tags. Moreover, these two approaches can be combined to yield even higher accuracy and efficiency.

This research is supported by National Science Foundation grant CNS-0716635 (Principal Investigator: Professor Gabriel Robins).
We have implemented, tested, and evaluated the proposed approach to confirm its general applicability, scalability, and reliability. Our approach suits a wide-range of requirements and tradeoffs including accuracy, speed, cost, and power. We have also identified several key challenges (e.g., environmental interferences, tag sensitivity, spatial arrangements of tags etc.) that adversely affect the performance of RFID-based object localization, and we propose mitigating techniques.

This paper is organized as follows. In section II, we describe related research efforts to localize mobile objects based on RFID technology. Several localization challenges and mitigating techniques are presented in section III. We present our localization approach in section IV, discuss implementation details and results in section V, and conclude in section VI with future research directions.

\section{RELATED WORK}

RFID-based localization for mobile objects can be broadly classified into tag and reader-based localization techniques, wherein position estimates of tags and readers attached to such objects are determined. In this paper, we focus on the localization of mobile objects by utilizing the far-field radiowave interaction between the RFID tags and readers (i.e., other RF-based localization approaches utilizing near-field, surface acoustic waves, microwaves, GPS, etc. are outside the scope of this work). Related research work includes the following.

Chae and Han [5] describe a two-step approach to localize mobile robots in an indoor environment. In their first step, an onboard RFID reader is coarsely localized with respect to neighborhood active reference tags. In the second step, a vision sensor combined with a feature detection algorithm identifies key environmental features to minimize the localization error to an average of 0.23 meters. Their approach is less applicable in different scenarios as the onboard vision sensor requires a sufficiently illuminated environment and objects must be within line-of-sight (a fundamental drawback that RFID was intended to eliminate in the first place).

Choi and Lee [8] propose to localize mobile robots in an indoor environment by utilizing ultrasonic sensors in combination with an onboard reader. In the first stage, the global position of the mobile robot is estimated through onboard reader localization with respect to the neighborhood passive reference tags. The second stage uses ultrasonic sensors for local position estimates. While their approach can yield higher accuracy, it is inherently not a pure RFID-based method, 
but rather a sound-based approach and is thus highly limited by issues such as environmental noise, line-of-sight, etc.

Hähnel et al [11] propose using a laser range scanner combined with an RFID reader onboard a mobile robot. The laser range scanner is used to learn a map comprised of reference tags, which in turn is used to estimate the position and orientation of mobile robots. However, this approach imposes line-of-sight constraints, and moreover tag orientation issues degrade the detection probability of the reference tags, resulting in high localization errors in the 1 to 10 meters range.

Han et al [12] propose mobile object localization by using reference tags and onboard mobile readers. Localization error is minimized using a triangular tag arrangement scheme, yielding average localization error of 0.09 meter in a small test region of one meter square. Koch et al [14] propose mobile object localization technique based on passive and active reference tags and onboard readers. Position estimates of the mobile objects can be determined within 0.1 meter accuracy on average.

Milella et al [18] utilize an onboard monocular camera, a reader and a tag bearing estimation technique based on "fuzzy inference system" to localize mobile robots. The average localization error is 0.64 meter. Senta et al [20] present a mobile robot localization technique based on reference tags, onboard readers, and a support vector machine (SVM)-based machine learning approach. This method yields localization errors of over 0.2 meters, and is limited by the spatial tag arrangement, measurement noise, and tag-reader proximity.

Seo and Lee [21] describe a mobile object localization system that transmits an RFID signal from an onboard reader to the neighborhood beacon, which in turn responds with an ultrasonic signal. The estimated distance is computed based on the time difference between transmitted and received signals, with an average localization error in the range of 0.2 to 1.6 meters. Vorst et al [23] present a mobile object localization approach using reference tags, onboard readers, and a particle filter-based technique. They compare prior-obtained training data with real-time RFID measurements to yield an average localization error in the range of 0.2 to 0.6 meters.

The effectiveness of the previous approaches is hindered by reliance on line-of-sight techniques, combining multiple nonRFID (e.g., ultrasonic sensors, cameras, lasers etc.) and RFID components in an ad-hoc manner, large numbers of onboard components, high localization delays, and heavy power requirements [5, 8, 11, 18]. Moreover, some of the above methods are too expensive or unwieldy due to the cost, size, and weight of the required infrastructure. Finally, the above approaches ignore the key issue that the RFID equipment itself can introduce significant amount of experimental errors. For example, previous works ignore the fact that identical tags can have widely varying detection sensitivities, which can greatly affect the experimental outcomes, as shown by Chawla, Robins, and Zhang [6]. Thus, instead of addressing and mitigating these basic principles (as we do in this paper), previous research efforts resort to Herculean efforts to reduce errors on other fronts, often resulting in a hodgepodge of adhoc and ineffectual techniques.

\section{LOCALIZATION CHALLENGES}

All RFID-based localization techniques have inherent position estimate errors due to various external (e.g., environmental) and internal (e.g., RFID tags and reader related) factors. This section describes key issues that induce localization errors and propose techniques to mitigate them.

\section{A. Interferrence and RF Occlusion}

Environmental factors such as radio noise and occlusions by liquids or metals can cause radio-wave scattering and attenuation, which can in turn result in localization errors. Mitigating techniques such as electrostatic shielding, full faraday cycle analysis, and path-loss contour mapping can help reduce the impact of such factors on localization accuracy [22]. Deploying more tags and readers in the experimental region can also reduce adverse interference and occlusion effects.

\section{B. Tag Sensitivity}

Tag detection sensitivity is characterized by the minimum power needed to read the tag at a particular distance. It is a function of chip threshold power sensitivity, tag antenna gain, and the chip's high impedance state [19]. Moreover, tag manufacturing variability can dramatically affect the detection sensitivities of tags. Thus, tags with low sensitivities become invisible at shorter distances than their higher-sensitivity counterparts, leading to position estimation errors. To address this issue, we propose a pre-processing step of sorting (i.e., "binning") the tags based on their detection sensitivities, and classify them as "highly sensitive", "average sensitive" and "low sensitive" using read measurements over different power and distance combinations [6]. This enables only uniformlysensitive tags to be deployed in the same experiment, resulting in more meaningful and consistent experimental results. Curiously, previous works all seem to ignore this critical issue.

\section{Tag Spatiality}

RFID-based mobile localization techniques typically utilize reference tags placed in known locations. The positions of these reference tags can significantly affect the localization accuracy, and regular placements of reference tags tend to yield lower positioning errors, as opposed to random arrangements.

\section{Tag Orientation}

Tag and reader interaction is significantly affected by the tag orientation. For example, Bolotnyy and Robins analyzed how tag orientation impacts the tag detection probability [3, 4]. In particular, they discovered that when multiple tags are placed on same object, orthogonal orientations yield much higher detection probabilities than parallel orientations.

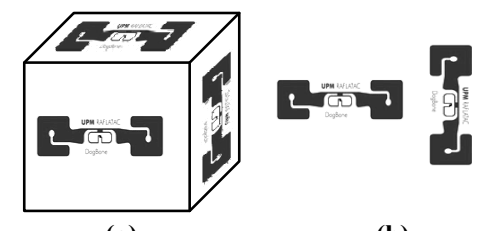

(a)

(b)

Figure 1. Tag orientations: (a) 3D orthogonal, (b) Planar orthogonal 
Figure 1(a), shows a 3D object with multiple orthogonally oriented tags, and Figure 1(b) shows an orthogonal planar (i.e., horizontal and vertical) orientations of two tags. In section IV, our experiments indicate that horizontal planar orientation increases tag sensitivity. Thus, utilizing multiple tags in orthogonal spatial and horizontal planar orientation improves localization accuracy.

\section{E. Reader Locality}

Theoretically, the RFID power-distance relationship is characterized based on the Friis transmission equation given as follows [7]:

$$
\frac{\mathrm{P}_{\mathrm{R}}}{\mathrm{P}_{\mathrm{T}}}=\mathrm{G}_{\mathrm{R}} \mathrm{G}_{\mathrm{T}}\left(\frac{\lambda}{4 \pi \mathrm{D}}\right)^{2}
$$

Here, $\mathrm{P}_{\mathrm{R}}$ is the power transmitted by the reader, $\mathrm{P}_{\mathrm{T}}$ is the power received at the tag, $\mathrm{G}_{\mathrm{R}}$ and $\mathrm{G}_{\mathrm{T}}$ are the antenna gain of the reader and the tag, $\lambda$ is the radio-wave wavelength, and $\mathrm{D}$ is the distance between the tag and reader. For a typical RFID system, the variables $\lambda, G_{R}$, and $G_{T}$ are the design parameters. Thus, by knowing the reader and tag power levels, the distance between them can be estimated. Alternatively, if the distance between the readers and tags are known, then the received power at the tags can be determined. Thus, the reader location impacts the localization accuracy. We propose that more tags should be placed in the region near the trajectory of mobile objects in order to improve the overall localization accuracy.

Our main principle behind above mitigating techniques is "to identify and minimize possible errors at the sources where they arise". This leads to efficient localization techniques, fewer onboard components, lower power requirements, and higher localization accuracy. In the following section, we use this principle to develop techniques for minimizing the mobile localization errors.

\section{MOBILE OBJect LOCALIZATION USING RFID}

The proposed localization approach utilizes two different techniques. In the first technique, an onboard reader and reference tags embedded in the environment are used to coarsely localize the mobile object. The second technique varies the power levels of environment-embedded readers to localize the onboard tag via the empirical power-distance relationship (calibrated using reference tags at known positions). To ensure uniform behavior from the tags, we test, sort, and select them on their (similar) detection sensitivity. Also, by employing multi-tags $[3,4]$, we reduce the uncertainties when inferring the position of onboard tags. Finally, we combine these localization techniques and propose several heuristics for significantly improving the localization accuracy.

While tags are sorted, placed, and calibrated as part of offline pre-processing phase, the actual localization and errorminimization heuristics are performed in real-time. By dividing the task of localization into separate phases, we reduce the time required to estimate positions of mobile objects. We describe key aspects of the proposed localization approach below.

\section{A. Calibrated Tags}

The accuracy of our localization approach relies on the tags having uniform detection sensitivities. Also, this property can help localization speed improve with higher tag sensitivities. Thus, as an offline pre-processing quality-control check, the sensitivities of all the tags are tested and characterized, to ensure that only tags with uniform (and high) sensitivities are used in our subsequent localization experiments. We have also developed a four-way multi-tag platform that provides higher operational reliability, as illustrated in Figure 2.
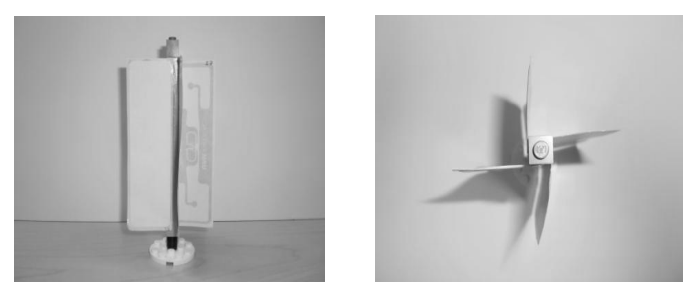

Figure 2. A four-way multi-tag platform

Figure 2 shows the design of our four-way multi-tag platform consisting of four "Impinj Dogbone Monza 3" UHF passive tags mounted on a vertical stand made of Lego bricks (our choice of Lego components is based on the versatility of Lego bricks as well as the transparency of their plastic material to radio-waves). We have built 33 such platforms, and each tag on the platform was calibrated separately using the techniques described by Chawla, Robins, and Zhang [6]. We have performed two types of platform calibration experiments to ensure uniform detection sensitivity across variables such as tag rotation and proximity, described as follows.

1) Proximity Sensitivity Calibration: In this experiment, we ensured that the four-way multi-tag platforms consisting of four proximate equally sensitive tags (Figure 2) all have similar sensitivities. This was achieved by determining the average read count of constituent tags having matching orientations with respect to the reader's antennas. Thus, tags at position one, two, three, and four were oriented towards antenna one, two, three, and four, respectively. We kept the reader power level constant at $31.6 \mathrm{dBm}$ and varied the distance between the reader and the multi-tags within the range of 1.27 to 3.81 meters.

We also varied the reader power level within the range of 25.6 to $31.6 \mathrm{dBm}$, keeping the distance between them constant at 2.54 meters. We repeated the calibration experiment three times and computed the average. While the combination of power level and distance range was comparatively small, variations in the tag sensitivities are evident at this scale. Figures 3(a), 3(b), and 3(c) illustrate the results of this experiment by varying the distance and keeping the reader power constant. For example, at a distance of 2.54 meters away from the reader, position four yields the highest average read counts. This is due to antenna four being nearer to the tag at position four than antenna one. Also, at 2.54 meters tag position three yields the lowest average read count, due to the shape of the RF signal lobe emitted by the antenna. Similar conclusions can be drawn from Figures 3(d), 3(e), and 3(f). 


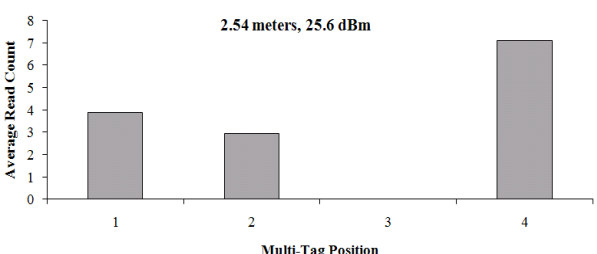

(a)

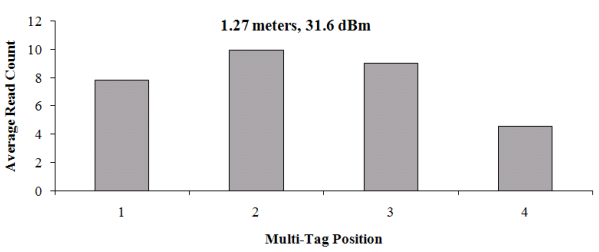

(d)

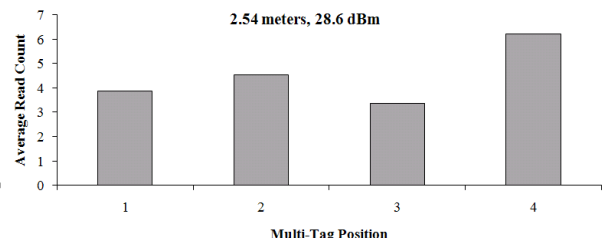

(b)

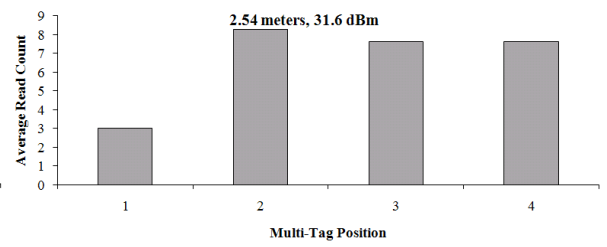

(e)

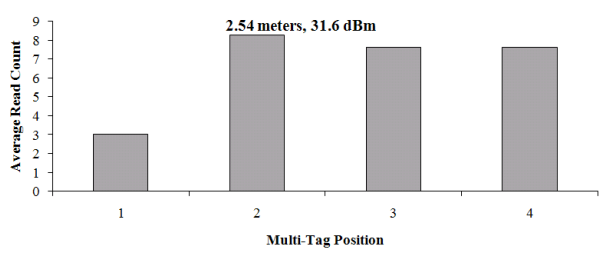

(c)

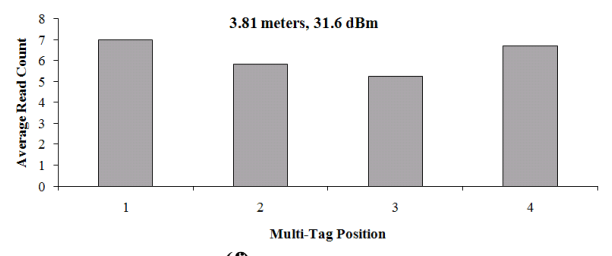

(f)

Figure 3. Multi-tag sensitivity measurements under proximity metric using constant-distance/variable-power and variable-distance/constant-power configurations.

2) Rotation Sensitivity Calibration: In this experiment, we determined the impact on tag sensitivity of rotating the multitag platforms. We varied the reader power level between 25.6 and $31.6 \mathrm{dBm}$ and kept the distance between the reader and the four-way multi-tag platform constant at 2.54 meters. Separately, we varied the distance between the reader and the four-way multi-tag platform within the range of 1.27 to 3.81 meters and kept the reader power level constant at $31.6 \mathrm{dBm}$. We then rotated each multi-tag platform counter-clockwise, repeating each calibration three more times and computed their average.

Figures 4(a)-4(1) and 5(a)-5(1) illustrate the impact of rotation on the average read-counts. In particular, each row of graphs depicts the average read count of four tags facing four antennas. When the platform is rotated counter-clockwise, these values are interchanged (e.g., tag at position one faces antenna one, and after a rotation, tag two takes that position and retains the read-count within permissible error range).

By combining the calibration results from the proximity and rotation experiments, it is evident that the 33 four-way multi-tags consisting of individually equally-sensitive tags are sensitivity invariant. This provides confidence that using these uniformly-sensitive multi-tags in subsequent localization experiments will help to minimize uncertainties due to tag variations, measurement noise, spatial orientations, etc.

\section{B. Localization Approach}

We now describe the proposed mobile object localization approach that consists of two different techniques based on the four localization algorithms. In the first technique, we localize readers onboard the mobile objects with respect to an environment instrumented with stationary reference four-way multi-tags. We measure the encountered unique tag IDs as the object moves around the environment. We associate a timestamp with each such measurement, resulting in a list of tuples of the type $\langle$ Tag ID, Timestamp $\rangle$. Thus, we determine the path of the mobile objects by knowing the location of reference tags and the measurement time. We call this algorithm "Measure and Report".

Mobile objects can be localized more accurately by using a regular arrangement of stationary reference tags. However, the limited read-range of the onboard reader, as well as the uncertainties in the actual locations of the reference tags, can introduce errors into the resulting position estimates. To minimize such errors, in our second technique we vary the power levels of the readers embedded in the environment in order to localize the target multi-tags onboard mobile objects using empirical power-distance relationships calibrated against reference tags. We provide three algorithms that control the reader power level in different ways, yielding tradeoffs between localization accuracy and overall speed.

In the first algorithm, we linearly increment the reader power level from lowest to highest in order to determine the minimum power level required to detect reference and onboard multi-tags. While this approach finds the minimum tag detection power levels, it may take more time to converge. Alternatively, we can instead vary the power level from highest to lowest in order to detect tags, since tags are typically not located near readers. Thus, stepping down the power level (i.e., from highest to lowest) will minimize the average number of iterations required to determine the minimum tag detection power level. We call this algorithm "Linear Search".

In the second algorithm, we start at a mid-value power level, and then either step-up or step-down based on the reader's ability to find the tags. Thus, we can converge faster on the minimum power level required for tag detection. We call this algorithm "Binary Search". Note that these two algorithms search for only one tag per execution cycle. Our third algorithm addresses this limitation by determining the minimum power levels of large groups of tags in parallel. Thus, it is equivalent to running a Linear Search algorithm in parallel for all the tags. This algorithm is called "Parallel Search". Since Parallel Search can determine the minimum power-levels of onboard tags in parallel, it enables the simultaneous localization of multiple mobile objects. 


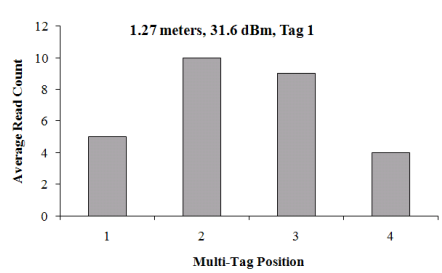

(a)

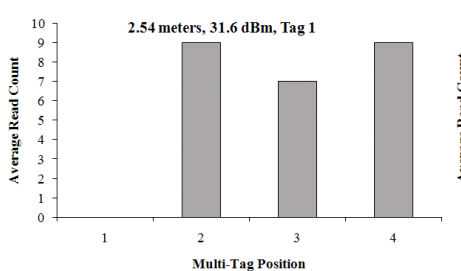

(e)

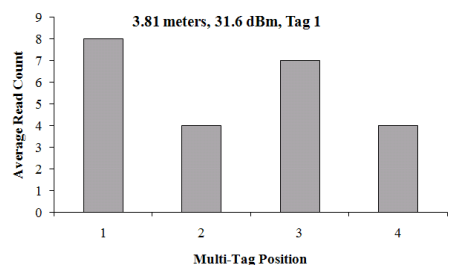

(i)

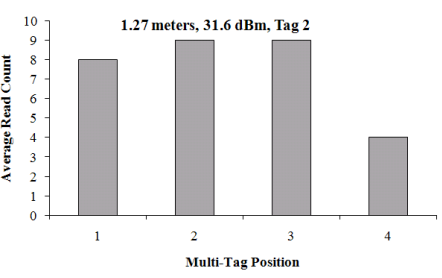

(b)

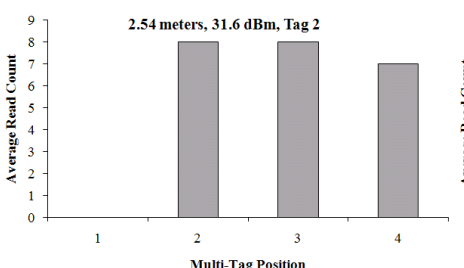

(f)

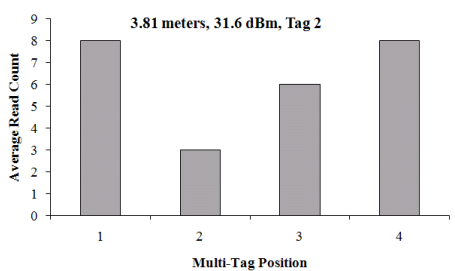

(j)

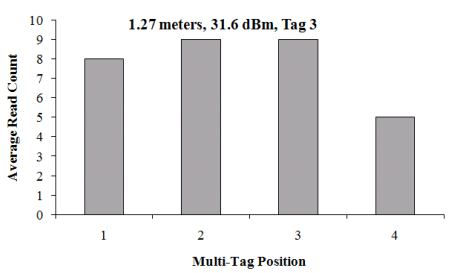

(c)

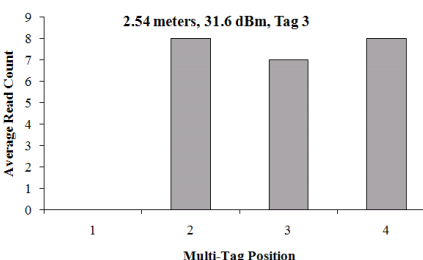

(g)

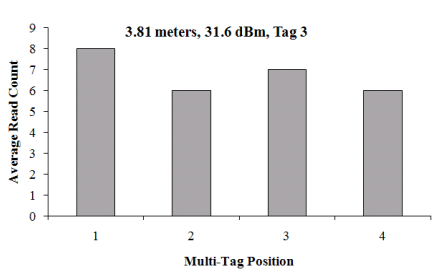

(k)

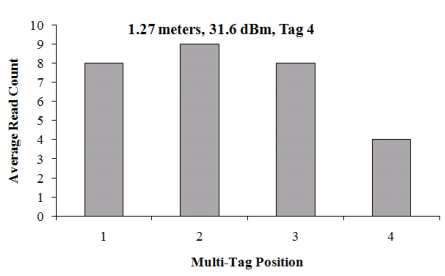

(d)

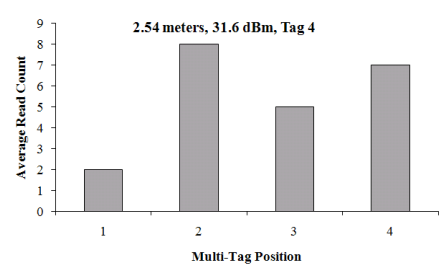

(h)

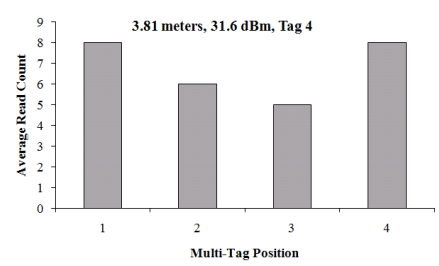

(l)

Figure 4. Multi-tag sensitivity measurements under rotation metric using variable-distance/constant-power configuration

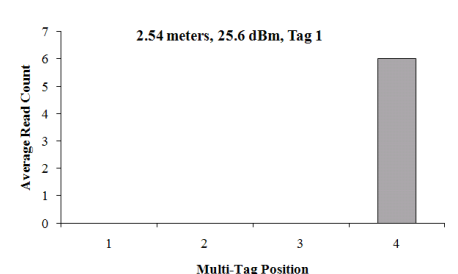

(a)

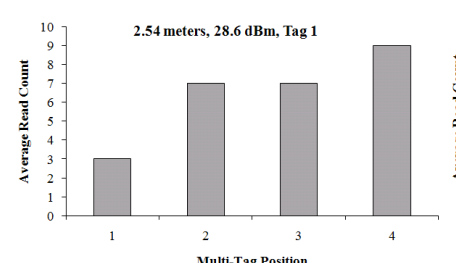

(e)

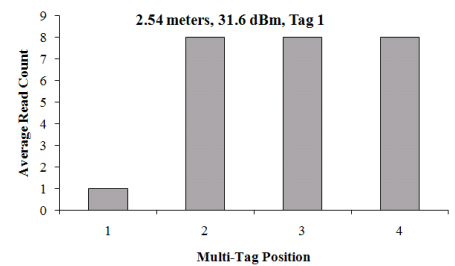

(i)

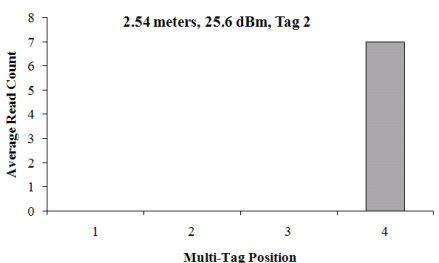

(b)

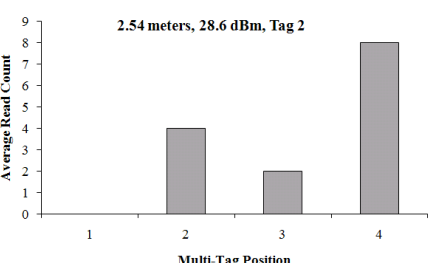

(f)

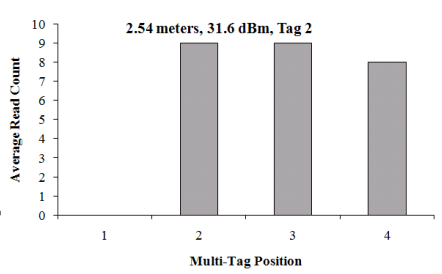

(j)

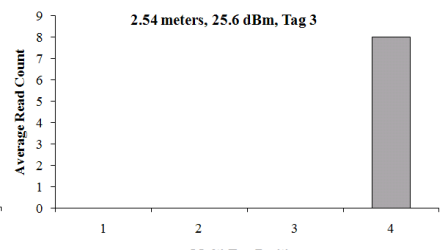

(c)

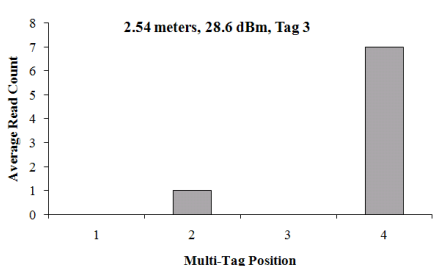

(g)

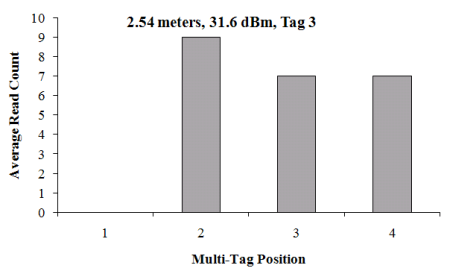

(k)

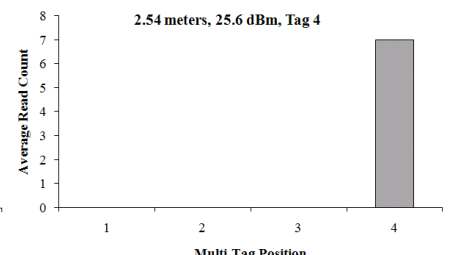

(d)

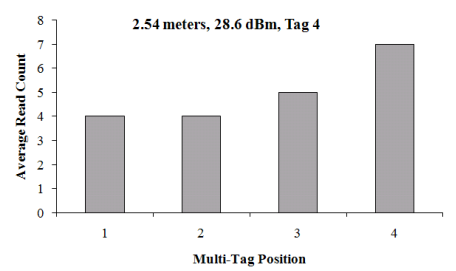

(h)

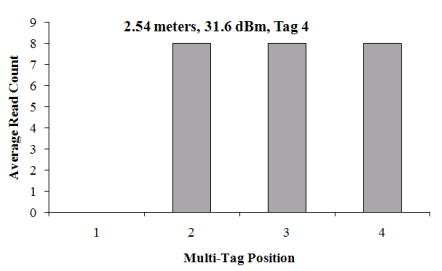

(I)

Figure 5. Multi-tag sensitivity measurements under rotation metric using constant-distance/variable-power configuration.

Table I gives the time complexity of each algorithm. While the Measure-and-Report algorithm is the fastest algorithm, both the Linear Search and Binary Search algorithms take considerably more time due to their operating in a serial manner. Since, the Parallel Search algorithm is independent of the number of tags and only dependent on the number of power 
levels, it requires less time than the Linear Search or Binary Search algorithms. Moreover, the algorithms that take more time tend to generate higher resolutions of the minimum power level required to detect tags. Alternatively, the faster algorithms trade off localization accuracy for speed.

TABLE I. TIME-COMPLEXITY OF LOCALIZATION ALGORITHMS

\begin{tabular}{|c|l|c|}
\hline $\begin{array}{c}\text { Localization } \\
\text { Technique }\end{array}$ & \multicolumn{1}{|c|}{$\begin{array}{c}\text { Localization } \\
\text { Algorithm }\end{array}$} & Time Complexity \\
\hline Reader Localization & Measure and Report & $\mathrm{O}(1)$ \\
\hline \multirow{3}{*}{ Tag Localization } & Linear Search [6] & $\mathrm{O}(\mathrm{N} \cdot \mathrm{P})$ \\
\cline { 2 - 3 } & Binary Search [6] & $\mathrm{O}(\mathrm{N} \cdot \log \mathrm{P})$ \\
\cline { 2 - 3 } & Parallel Search [6] & $\mathrm{O}(\mathrm{P})$ \\
\hline
\end{tabular}

$\mathrm{N}=$ Number of tags, $\mathrm{P}=$ Number of reader power levels used

We can utilize these algorithms in different combinations to trade off localization accuracy, speed and power requirements. Furthermore, localization errors can occur due to (1) the onboard reader operating range, (2) implicitly identifying the four-way multi-tag platforms with the nearest reference tags, and (3) the inherent minimum power level estimation errors of the algorithms. We discuss these errors along with mitigating techniques below.

\section{Localization Error and Heuristics}

Localization errors occur in the first technique (i.e., onboard reader localization) due to limitations in the power and read-range of the onboard reader. Since mobile objects can move arbitrarily, an inexpensive and reliable way to reduce this type of error is by placing more densely/regularly arranged reference tags in the nearby region. In the second technique (i.e., onboard tag localization), errors in position estimates occur by identifying the onboard multi-tag with the nearest neighborhood reference tags.

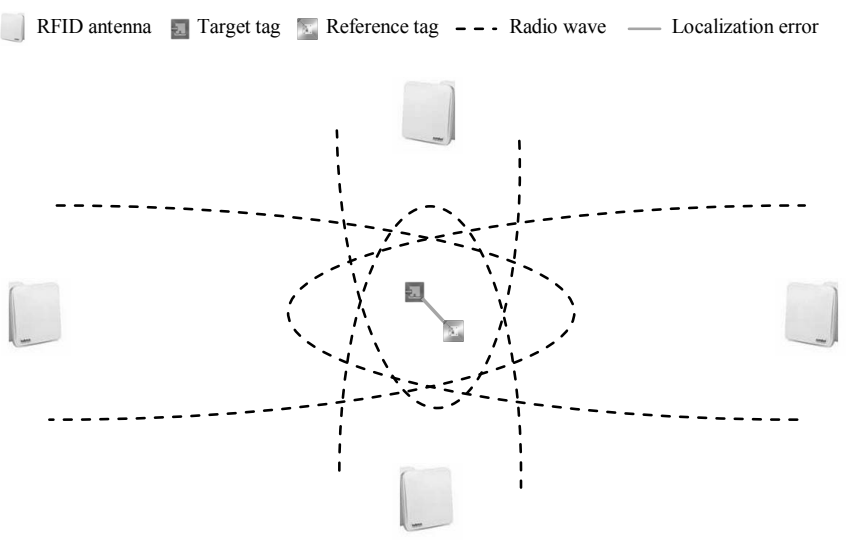

Figure 6. Sources of localization errors.

Figure 6 depicts four antennas emitting radio waves forming an intersection region where onboard target tags (i.e., four-way multi-tag platforms) are likely to be present. This intersection region gives coarse-level position estimates of the four-way multi-tag platforms, which is further refined by using the positions of nearby reference tags to estimate the positions of multi-tags. However, this can lead to potential "round off" errors. In order to minimize possible localization errors, we have developed eleven heuristics that utilize the differences in the reader power levels, the orthogonal positions of the readers, and the neighborhood reference tags. We describe these error mitigation heuristics in detail below.

TABLE II. LOCALIZATION ERROR HEURISTICS

\begin{tabular}{|l|l|}
\hline \multicolumn{1}{|c|}{ Error Heuristic } & \multicolumn{1}{c|}{ Description } \\
\hline Absolute Difference [6] & $\begin{array}{l}\text { Compute the absolute difference of the reader power } \\
\text { levels between the neighborhood and onboard multi- } \\
\text { tags. There are four such heuristics. }\end{array}$ \\
\hline $\begin{array}{l}\text { Minimum Power } \\
\text { Reader Selection [6] }\end{array}$ & $\begin{array}{l}\text { Compute the absolute difference of the power levels } \\
\text { between the neighborhood and onboard multi-tags } \\
\text { using the minimum power levels of the two orthogonal } \\
\text { readers. There are two such heuristics. }\end{array}$ \\
\hline $\begin{array}{l}\text { Root Sum Square } \\
\text { Absolute Difference [6] }\end{array}$ & $\begin{array}{l}\text { Compute the square root of the sum of squares of the } \\
\text { absolute difference of the reader power levels between } \\
\text { the neighborhood and onboard multi-tags. There are } \\
\text { four such heuristics. }\end{array}$ \\
\hline Meta-Heuristic [6] & $\begin{array}{l}\text { Compute the minimum over all power levels obtained } \\
\text { using above the heuristics. Thus, it is a meta-heuristic } \\
\text { that enables minimum tag detection power levels. }\end{array}$ \\
\hline
\end{tabular}

\section{EXPERIMENTS AND RESULTS}

This section presents the implementation details, evaluation methodology, and experimental results pertaining to the proposed mobile object localization approach. Furthermore, we compare the proposed localization approach with existing mobile object localization techniques. Our experiments were performed in an indoor environment using one onboard reader and one four-way multi-tag platform per mobile object. Also, one stationary reader, four antennas, and 33 reference tags were embedded in the surrounding environment. Table III describes the experimental setup and implementation details.

TABLE III. EXPERIMENTAL SETUP DETAILS

\begin{tabular}{|c|c|c|c|c|}
\hline Type & \multicolumn{4}{|c|}{ Technology Parameters } \\
\hline \multirow{3}{*}{ Workstation } & $C P U$ & $\begin{array}{l}\text { AMD Athlon } \\
64 @ 2 \mathrm{GHz}\end{array}$ & OS & WinXP \\
\hline & $R A M$ & 1 GBytes & $P L$ & $\mathrm{C}++/ \mathrm{C} \#$ \\
\hline & Hard Disk & 100 GBytes & $A P I$ & M4 LIB \\
\hline \multirow{3}{*}{$\begin{array}{c}\text { RFID } \\
\text { Equipment }\end{array}$} & $\begin{array}{l}\text { Reader } \\
\text { Type }\end{array}$ & $\begin{array}{l}\text { ThingMagic } \\
\text { M4 iDtronic } \\
\text { Voltaire CF }\end{array}$ & Protocol & $\begin{array}{l}\text { EPC } \\
\text { Gen2 }\end{array}$ \\
\hline & Antenna & $\begin{array}{l}\text { Linear with } \\
\text { 6dBi gain }\end{array}$ & $\begin{array}{l}\text { Reader } \\
\text { Devices }\end{array}$ & 3 \\
\hline & Tag & $\begin{array}{c}\text { Impinj } \\
\text { Dogbone } \\
\text { Monza } 3 \\
93 \times 23 \mathrm{~mm}\end{array}$ & Antennas & 6 \\
\hline \multirow[b]{2}{*}{ Environment } & Map Area & $8 \mathrm{~m}^{2}$ & \multirow[b]{2}{*}{$\begin{array}{l}\text { Reference } \\
\text { Multi-Tags }\end{array}$} & \multirow[b]{2}{*}{33} \\
\hline & $\begin{array}{l}\text { Room } \\
\text { Volume }\end{array}$ & $41 \mathrm{~m}^{3}$ & & \\
\hline Robots & Kit & $\begin{array}{c}\text { Lego } \\
\text { Mindstorms }\end{array}$ & Robots & 2 \\
\hline Onboard Control & Model & $\begin{array}{c}\text { HP iPAQ } \\
\mathrm{h} \times 2490\end{array}$ & $P D A s$ & 2 \\
\hline Onboard Link & Type & Bluetooth & $\begin{array}{l}\text { Bluetooth } \\
\text { Dongles }\end{array}$ & 2 \\
\hline
\end{tabular}

We developed two mobile robots using Lego Mindstorm kits. Figure 7(a) illustrates the mobile robots with their onboard controller consisting of one HP iPAQ hx2490, one iDtronic Voltaire portable RFID reader, and one four-way multi-tag platform. Figure 7(b) depicts our experimental railroad track for operating these mobile robots. Also, shown are the four- 
way multi-tag platforms used as the reference tags. We coarsely localize the mobile objects by utilizing the onboard reader to read the reference tags encountered during motion and transmit the tag IDs to the backend workstation using the onboard bluetooth link. At any location we can further refine the coarse-level position estimates by varying the stationary reader power levels to localize the onboard multi-tags.

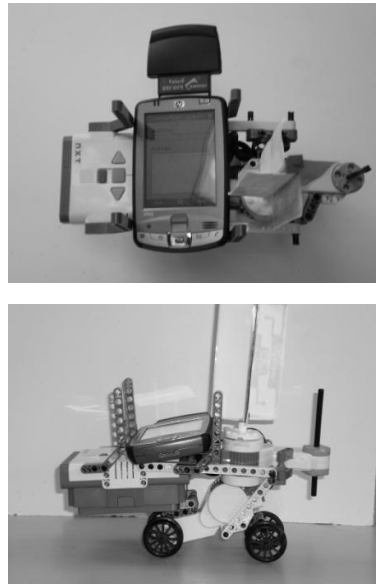

(a)

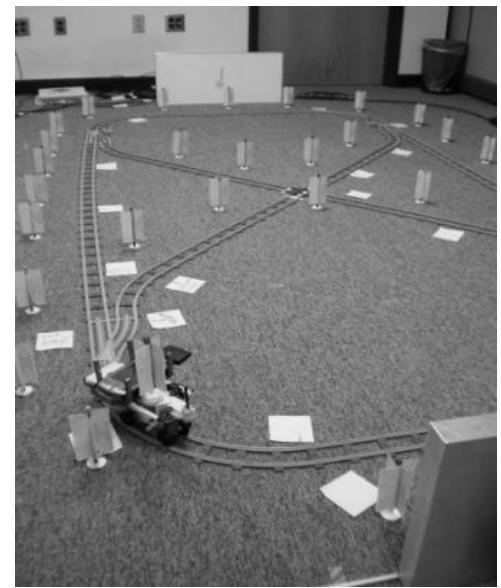

(b)
Figure 7. Experiment components: (a) Mobile robot platform, and (b) Track

Note that approaches that utilize only RFID technology for precise localization impose speed limits on the moving objects due to the delays inherent in determining the minimum tag detection power levels, and the reader's operational speed. Thus, while coarser position estimates can be obtained for tags moving at several meters per second, slower speeds are still necessary for more precise localization. Such locomotion speed limitations are also present in other existing RFID-based mobile object localization techniques.

A key aspect of the proposed localization approach is to limit/eliminate the use of onboard non-RFID components, while still obtaining good localization accuracy and speed. This approach enables low on-board power consumption, as well as reduced overall cost and complexity. Our localization apparatus uses a single rechargeable $1440 \mathrm{mAh}$ Lithium-Ion battery for the onboard PDA control, RFID reader and bluetooth link. Thus, by reducing the needed onboard components, we reduce the power requirements of the mobile objects. Furthermore, to improve localization speed, we divide the proposed localization approach into a setup phase and a localization phase, thus decoupling the initial time-consuming calibration process from the subsequent localizations of the mobile objects.

During the setup phase, we distributed 33 reference tags across a 2D region and calibrated their empirical powerdistance relationships. In the localization phase, we estimated positions by using the proposed localization approach. For each phase, we utilized the localization algorithms in different combinations in order to minimize localization errors. We used the Linear Search algorithm in the setup phase, while the Binary Search algorithm was used in the localization phase, combined with the Measure and Report algorithm. Figures 8(a) and 8(b) illustrate the localization accuracy independently along the $\mathrm{X}$ and $\mathrm{Y}$-axis. Our data confirms that along both axes the proposed localization approach closely approximates the actual mobile robot positions.

Also, we hypothesized that increasing the number of reference tags increases the localization accuracy only up to a certain point. To test this hypothesis, we varied the number of references tags from 1 to 33 and determined that the range of localization error varied from 1.2 to 0.2 meters, as depicted in Figure 8(c). Thus, adding additional inexpensive passive reference tags increases the localization accuracy only up to a point (e.g., with only 22 reference tags, $88 \%$ of the maximum possible localization accuracy was achieved). Finally, we compared the proposed localization approach with existing localization techniques, as summarized in table IV.

TABLE IV. COMPARISON OF PROPOSED APPROACH WITH EXISTING RFIDBASED MOBILE LOCALIZATION TECHNIQUES

\begin{tabular}{|c|c|c|c|c|}
\hline \multirow[b]{2}{*}{ Technique } & \multicolumn{2}{|c|}{ Average Time (min) } & \multirow{2}{*}{$\begin{array}{l}\text { Test } \\
\text { area } \\
\left(\mathbf{m}^{2}\right)\end{array}$} & \multirow[b]{2}{*}{$\begin{array}{c}\text { Localization } \\
\text { Error (m) }\end{array}$} \\
\hline & $\begin{array}{l}\text { Setup } \\
\text { Phase }\end{array}$ & $\begin{array}{c}\text { Localization } \\
\text { Phase }\end{array}$ & & \\
\hline Chae and Han [5] & NR & NR & 48.36 & 0.23 \\
\hline Choi and Lee [8] & NR & NR & 14.4 & $0.016-0.024$ \\
\hline Hähnel et al [11] & NR & NR & 784 & $1-10$ \\
\hline Han et al [12] & NR & NR & 1 & 0.09 \\
\hline Koch et al [14] & NR & NR & 60 & 0.1 \\
\hline Milella et al [18] & NR & NR & 70 & 0.64 \\
\hline Senta et al [20] & NR & NR & 2 & 0.2 \\
\hline Seo and Lee [21] & NR & NR & 5 & $0.2-1.6$ \\
\hline Vorst et al [23] & NR & NR & 125 & $0.2-0.6$ \\
\hline $\begin{array}{c}\text { Proposed } \\
\text { Localization } \\
\text { Approach: (Alg. IA, } \\
\text { IB, II, III, IV) }\end{array}$ & $\begin{array}{c}29.78 \\
161.23 \\
47.24 \\
1.67 \\
0\end{array}$ & $\begin{array}{l}1.42, \\
5.28, \\
1.95, \\
1.67, \\
0\end{array}$ & 8 & $\begin{array}{c}0-0.9 \\
\text { Avg. }=0.23\end{array}$ \\
\hline
\end{tabular}

II - Binary Search (Low to High); Alg. III - Parallel Search; and Alg. IV - Measure and Report

The maximum time required by the proposed localization approach in the setup phase is due to the Linear Search algorithm operating in low-to-high mode. While this setup

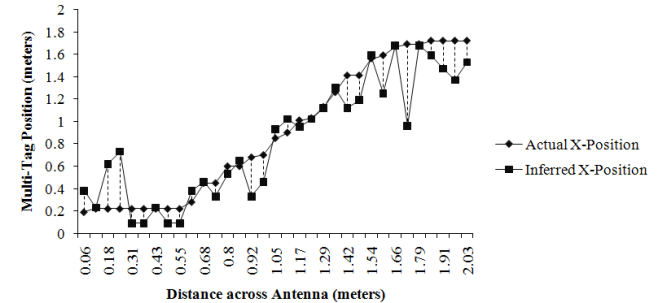

(a)

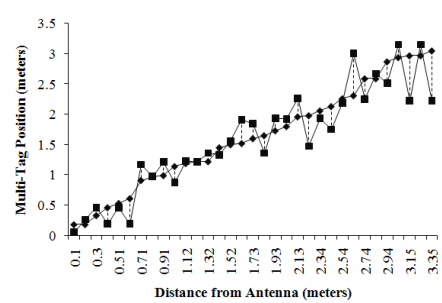

(b)
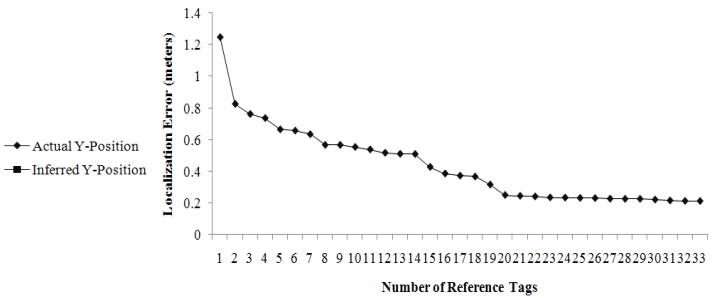

(c)

Figure 8. Localization accuracy: (a) Along the X-axis, (b) Along the Y-axis, and (c) Impact of the number of reference tags on localization accuracy 
time is not trivial, the setup phase is an offline pre-processing step that is invoked only infrequently. We note that tags are susceptible to changes in the operating environment (e.g., ambient temperature and humidity, presence of metals and liquids, etc.). Therefore, repeating the tag binning process and the setup phase occasionally can ensure that consistently high localization accuracy is achieved and maintained. To aid the end-user with the real-time tracking and visualization of the target mobile objects, we have developed a GUI-based mapping application, as shown in Figure 9. Using this application, the user can experiment with different parameters (e.g., number of antennas, combinations of localization algorithms, error heuristics etc.) and study their impact on the overall localization accuracy and speed.

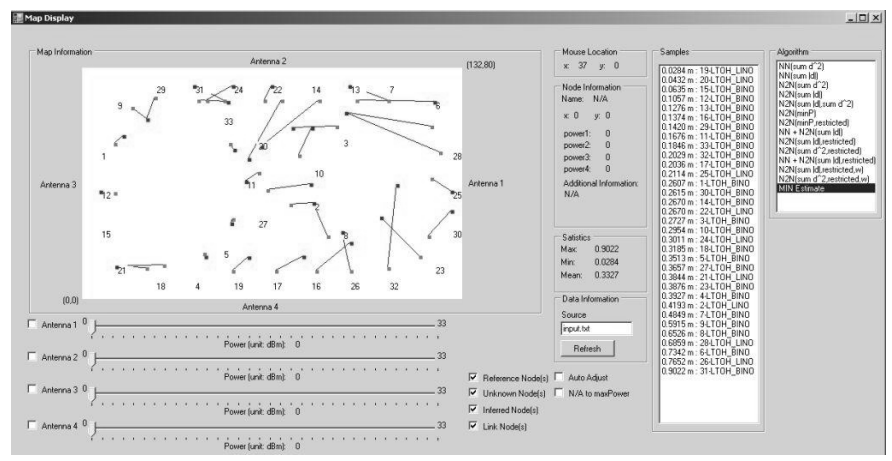

Figure 9. Graphical visualization interface for the RFID localization system.

\section{CONCLUSION}

In this paper, we have proposed an accurate, scalable, reliable, power-efficient approach to localize mobile objects. We have outlined several localization challenges and proposed practical techniques to mitigate them. We deployed uniformlysensitive four-way multi-tag platforms in order to improve the mobile object localization accuracy and speed. Furthermore, we analyzed the impact of the number of reference tags on the localization accuracy, and compared the proposed approach to existing localization approaches in terms of accuracy, speed, and power consumption. While these experiments were performed in an indoor setting, the proposed localization approach is general enough to be widely applicable (e.g., to 3D regions, outdoor environments, multiple stationary and mobile object localization, etc.). Future research can also strive for further improvements in the overall localization speed.

\section{REFERENCES}

[1] G. D. Abowd, and E. D. Mynatt, "Charting Past, Present, and Future Research in Ubiquitous Computing", ACM Transactions on ComputerHuman Interaction, ACM Press, Vol. 7, Issue 1, Mar. 2000, pp. 29-58.

[2] G. Blewitt, C. Kreemer, W. C. Hammond, H. Plag, S. Stein, and E. Okal, "Rapid Determination of Earthquake Magnitude using GPS for Tsunami Warning Systems", Geophysical Research Letters, American Geophysical Union, Vol. 33, L11309, Jun. 2006, 4 pages.

[3] L. Bolotnyy, and G. Robins, "The Case for Multi-Tag RFID Systems", IEEE International Conference on Wireless Algorithms, Systems and Applications (WASA 2007), Chicago, Aug. 2007, pp. 174-186.

[4] L. Bolotnyy, and G. Robins, "Multi-Tag RFID systems", Security in RFID and Sensor Networks, Auerbach Publications, CRC Press, Taylor \& Francis Group, 2009, pp. 3-28.
[5] H. Chae, and K. Han, "Combination of RFID and Vision for Mobile Robot Localization", IEEE International Conference Intelligent Sensors, Sensor Networks and Information Processing (ISSNIP 2005), Melbourne, Australia, Dec. 2005, pp. 75-80.

[6] K. Chawla, G. Robins, and L. Zhang, "Object Localization using RFID", IEEE International Symposium of Wireless and Pervasive Computing (ISWPC 2010), Modena, Italy, May 2010, pp. 301-306.

[7] K. Finkenzeller, "RFID-Handbook: Fundamentals and Applications in Contactless Smart Cards and Identification", Second Edition, Munich, Germany: Wiley and Sons Inc., 2003.

[8] B. Choi, and J. Lee, "Mobile Robot Localization Scheme Based on RFID and Sonar Fusion System", IEEE International Symposium on Industrial Electronics (ISIE 2009), Seoul, South Korea, Aug. 2009, pp. 1035-1040.

[9] D. Estrin, D. Culler, K. Pister, and G. Sukhatme, "Connecting the Physical World with Pervasive Networks", IEEE Pervasive Computing, IEEE Press, Vol. 1, Issue 1, Jan. 2002, pp. 59-69.

[10] P. Fontelo, M. Ackerman, G. Kim, and C. Locatis, "The PDA as a Portal to Knowledge Sources in a Wireless Setting", Mary Ann Liebert Inc. Publishers, Telemedicine Journal and e-Health, Vol. 9, No. 2, Jun. 2003, pp. 141-147.

[11] D. Hähnel, W. Burgard, D. Fox, K. Fishkin, and M. Philipose, "Mapping and Localization with RFID Technology", IEEE International Conference on Robotics and Automation (ICRA 2004), Los Angeles, Jun. 2004, pp. 1015-1020.

[12] S. Han, H. Lim, and J. Lee, "An Efficient Localization Scheme for a Differential-Driving Mobile Robot Based on RFID System", IEEE Transactions on Industrial Electronics, IEEE Press, Vol. 54, Issue 6, Dec. 2007, pp. 3362-3369.

[13] J. Hightower, and G. Borriello, "Location Systems for Ubiquitous Computing", IEEE Computer, IEEE CS Press, Vol. 34, Issue 8, Aug. 2001, pp. 57-66.

[14] J. Koch, J. Wettach, E. Bloch, and K. Berns, "Indoor Localization of Humans, Objects, and Mobile Robots with RFID Infrastructure", IEEE International Conference on Hybrid Intelligent Systems (HIS 2007), Kaiserslautern, Germany, Sept. 2007, pp. 271-276.

[15] X. Liu, M. Corner, and P. Shenoy, "Ferret: RFID Localization for Pervasive Multimedia", Lecture Notes in Computer Science, Berlin, Germary, Springer Press, Sep. 2006, Vol. 4206/2006, pp. 422-440.

[16] F. Mattern, "The Vision and Technical Foundations of Ubiquitous Computing", UPGRADE - The European Online Magazine for the IT Professional, Vol. 2, No. 5, Oct. 2001, 6 pages.

[17] R C. Merrell, N. Merriam, and C. Doarn, "Information Support for the Ambulant Health Worker", Mary Ann Liebert Inc., Publishers, Telemedicine Journal and e-Health, Vol. 10, No. 4, Feb. 2005, pp. 432436.

[18] A. Milella, D. Di Paola, G. Cicirelli, and T. D'orazio, "RFID Tag Bearing Estimation for Mobile Robot Localization", IEEE International Conference on Advanced Robotics (ICAR 2009), Munich, Germany, Jul. 2009, pp. 1-6.

[19] P. V. Nikitin, and K.V.S. Rao, "Antennas and Propagation in UHF RFID Systems", IEEE International Conference on RFID (RFID 2008), Nevada, 2008, pp. 277-288.

[20] Y. Senta, Y. Kimuro, S. Takarabe, and T. Hasegawa, "Machine Learning Approach to Self-Localization of Mobile Robots using RFID Tag", IEEE/ASME International Conference on Advanced Intelligent Mechantronics, Zurich, Switzerland, Dec. 2007, pp.1-6.

[21] D. Seo, and J. Lee, "Localization Algorithm for a Mobile Robot using iGS", International Federation of Automatic Control - World Congress, Reed Elsevier Publishing Corp., Vol. 17, Part 1, pp. 742-747.

[22] Patrick J. Sweeney, "RFID for Dummies", Wiley Publishing Inc., 2005.

[23] P. Vorst, S. Schneegans, B. Yang, and A. Zell, "Self-Localization with RFID Snapshots in Densely Tagged Environments", IEEE/RSJ International Conference on Intelligent Robots and Systems (IROS 2008), Nice, France, Sep. 2008, pp. 1353-1358.

[24] R. Want, "RFID - A Key to Automating Everything", Scientific American, 2004, pp. 56-65. 